\title{
Learning with Small Data
}

\author{
Zhenhui Li \\ Pennsylvania State University \\ State College, Pennsylvania \\ JessieLi@psu.edu
}

\author{
Huaxiu Yao \\ Pennsylvania State University \\ State College, Pennsylvania \\ huaxiuyao@psu.edu
}

\author{
Fenglong Ma \\ Pennsylvania State University \\ State College, Pennsylvania \\ fenglong@psu.edu
}

\begin{abstract}
In the era of big data, it is easy for us collect a huge number of image and text data. However, we frequently face the real-world problems with only small (labeled) data in some domains, such as healthcare and urban computing. The challenge is how to make machine learn algorithms still work well with small data? To solve this challenge, in this tutorial, we will cover the state-of-the-art machine learning techniques to handle small data issue. In particular, we focus on the following three aspects: (1) Providing a comprehensive review of recent advances in exploring the power of knowledge transfer, especially focusing on meta-learning; (2) introducing the cuttingedge techniques of incorporating human/expert knowledge into machine learning models; and (3) identifying the open challenges to data augmentation techniques, such as generative adversarial networks. We believe this is an emerging and potentially highimpact topic in computational data science, which will attract both researchers and practitioners from academia and industry.
\end{abstract}

\section{CCS CONCEPTS}

-Information systems $\rightarrow$ Data mining; • Computing methodologies $\rightarrow$ Machine learning;

\section{KEYWORDS}

Meta-learning, transfer learning, knowledge regularization, generative models

\section{ACM Reference Format:}

Zhenhui Li, Huaxiu Yao, and Fenglong Ma. 2020. Learning with Small Data. In The Thirteenth ACM International Conference on Web Search and Data Mining (WSDM'20), February 3-7, 2020, Houston, TX, USA. ACM, New York, NY, USA, 4 pages. https://doi.org/10.1145/3336191.3371874

\section{INTENDED AUDIENCE}

All the researchers and practitioners are welcome. The audiences are assumed to have basic knowledge in linear algebra and machine learning. In particular, those who have engaged in related research and applications domains, e.g., supervised learning, semisupervised learning, active learning, deep learning, meta learning, optimization, etc., will be encouraged to have Q\&A interaction during the tutorial or further discussions offline. This tutorial aims to be composed of a good balance between the introductory and

Permission to make digital or hard copies of part or all of this work for personal or classroom use is granted without fee provided that copies are not made or distributed for profit or commercial advantage and that copies bear this notice and the full citation on the first page. Copyrights for third-party components of this work must be honored.

For all other uses, contact the owner/author(s).

WSDM '20, February 3-7, 2020, Houston, TX, USA

(C) 2020 Copyright held by the owner/author(s).

ACM ISBN 978-1-4503-6822-3/20/02.

https://doi.org/10.1145/3336191.3371874 advanced material (50\% for beginners and $50 \%$ for intermediate and advanced).

\section{PREVIOUS OFFERING OF THE TUTORIAL}

The topic of this tutorial is relatively new to machine learning and data mining area, and this is the first tutorial to systematically focus on small data. We have offered this tutorial internally at Penn State (https://sites.psu.edu/daml/) and have drawn a full class of students and have received very positive feedback.

\section{PRESENTERS}

\subsection{Contacts of the Presenters}

Zhenhui (Jessie) Li (main contact), Email: JessieLi@psu.edu, Tel: 814-863-6317, Website: https://faculty.ist.psu.edu/jessieli/

Huaxiu Yao, Email: huaxiuyao@psu.edu, Tel: 814-862-8330, Website: http://huaxiuyao.mystrikingly.com/

Fenglong Ma, Email: fenglong@psu.edu, Tel: 814-863-9497, Website: http://www.personal.psu.edu/ffm5105/

\subsection{Full Biography of the Presenters}

Dr. Zhenhui Li is a tenured associate professor of Information Sciences and Technology at the Pennsylvania State University. She is Haile family early career endowed professor. Prior to joining Penn State, she received her PhD degree in Computer Science from University of Illinois Urbana-Champaign in 2012, where she was a member of data mining research group. Her research has been focused on mining spatial-temporal data with applications in transportation, ecology, environment, social science, and urban computing. She is a passionate interdisciplinary researcher and has been actively collaborating with cross-domain researchers. She has served as organizing committee or senior program committee of many conferences including KDD, ICDM, SDM, CIKM, and SIGSPATIAL. She has been regularly offering classes on data organizing and data mining since 2012 . Her classes have constantly received high student ratings. She has received NSF CAREER award, junior faculty excellence in research, and George J. McMurtry junior faculty excellence in teaching and learning award.

Huaxiu Yao is currently a Ph.D. candidate of College of Information Sciences and Technology at the Pennsylvania State University. He got his B.Eng. degree from the University of Electronic Science and Technology of China. His research interests focus on developing cost-efficient machine learning algorithms with the applications in urban computing, graph analysis and computer vision. He has published over 10 papers on top conferences and journals such as ICML, KDD, AAAI, WWW and TIST. He has served as a program committee member in major machine learning and data mining conferences such as ICML, ICLR, AAAI, CIKM. 
Dr. Fenglong Ma is currently an assistant professor of the College of Information Sciences and Technology at Pennsylvania State University. He received his $\mathrm{PhD}$ degree from the Department of Computer Science and Engineering, the State University of New York at Buffalo in 2019, and subsequently joined Pennsylvania State University. His research interests lie in data mining and machine learning, with an emphasis on mining health-related data. His research interests also include Crowdsourcing, Internet of Things, Social Network Mining and Security. He has published over 40 papers in top conferences and journals such as KDD, WWW, CIKM, WSDM, ICDM, SDM, ACL, IJCAI, MobiCom, INFOCOM and TKDE. He presented a 3-hour tutorial at KDD 2019, and the title is "Optimizing the Wisdom of the Crowd: Inference, Learning, and Teaching".

\section{MOTIVATION}

In the real-world application, we often face the challenge with only small labeled data. For example, to detect outliers, we have small labeled outlier data; to detect trending news topics, the labeled topics are at a small number in early age; to diagnose a rare disease, the number of similar cases is small; to predict emerging events in a city, the chance of similar emergent events happened before is small. However, those small data are often the cases we care the most and we hope the model to be able to learn efficiently even with only small training samples.

In the field of computer vision and robotics, few-shot learning is a trending topic [39]. The new techniques that draw increasing attention include generative adversarial networks (GAN) [9] and meta learning $[6,30,37]$. Those techniques, on the one hand, still have room for improvement; on the other hand, they have not been yet widely applied to other real-world applications besides computer vision and robotics. The differences lie in the data structures and problem-specific settings. In addition, in many real-world applications, domain expert knowledge becomes very important and we cannot simply reply on black-box machine learning models.

To deal with small data issue in the real-world applications, we propose to give this tutorial so we can systematically study following perspectives: (1) the classical techniques such as semisupervised learning, active learning, and multi-task learning; (2) trending few-shot techniques in computer vision such as GAN; (3) trending few-short learning techniques in robotics such as meta learning; and (4) new techniques in incorporating domain knowledge.

\section{OUTLINE OF THE TOPICS TO BE COVERED}

\subsection{Part I: Transfer knowledge from models}

In this part, we address scenario where we have small labeled training in the target domain but can borrow the knowledge learned from other tasks in the source domain. In particular, we would like to cover the following topics:

- Transfer learning. In the setting of traditional transfer learning, there is only one task in the source domain, and another one task in the target domain.

- We will first discuss the scenario where the target domain has some labeled data. The most typically used technique is fine tuning technique.
- We then discuss the scenario where the target domain has no labeled data, which is the unsupervised transfer learning/domain adaptation technique.

* Discrepancy-based method defines the loss function by considering the discrepancy between source and target $[21,23]$.

* Adversarial method adds a new loss to differentiate a data sample generated by source domain or target domain $[20,35,36]$.

- Multi-task Learning. In the multi-task setting, we have $n$ tasks in the source domain, and the same $n$ tasks in the target domain. Typical approach includes defining the loss function as a combination of losses of all tasks [22, 28].

- Meta-learning. In the meta-learning setting, we have $n$ tasks in the target domain, and a new task in the target domain. This technique has recently shown to be very effective.

- Gradient-based method tries to find a better optimization process for the new task in the target domain by learning from the gradients of the tasks in the source domain. Representatively, Model-Agnostic Meta-Learning (MAML) and its variants[5-7, 18, 45] learn a optimal parameter initialization and have been applied to different fields, such as image classification [5], machine translation [11], spatiotemporal analysis [41, 48], AutoML [40] and graph analysis [32].

- Non-parametric methods adopt lazy learner (e.g., kNN) and learns the prototypes of the tasks in the source domain [27, 30, 37, 43, 46, 49].

- When learning from the tasks in the source domain, the tasks could be heterogeneous and simply "averaging" the knowledge of those tasks could hurt the performance by misleading the target domain task. In this topic, we further survey the recent work in addressing task heterogeneity $[38,42]$.

\subsection{Part II: Transfer knowledge from domain expert}

In this part, to address small data issue, we hope to borrow the knowledge from external domain experts. The key challenge lies in how to incorporate the knowledge in a different format into the learning models.

- One approach is to enriching representations using knowledge graph. Examples include using ConceptNet knowledge graph [31] for text classification and using health domain knowledge graph for health diagnosis [4, 25, 26, 44].

- Another approach is to add constraints in the loss function by incorporating domain knowledge. Traditional approach in Bayesian could be directly adding the prior. In discriminative model, we can add the constraint in the loss function [24].

- Though it is easy to obtain enough training data in natural language processing domain, there are many studies focusing on incorporating knowledge graph to improve performance $[12,14,19,34,50]$. We also briefly review such work. 


\subsection{Part III: Data augmentation}

In the last part, we will cover data augmentation techniques. When we have small labeled training data, we can try to add more training data by generating the labeled data through training data or through testing data or both.

- We will first discuss how to augment the data using labeled data.

- One commonly used traditional approach is to generate more training data by hand-crafted rules. These methods are usually specific to certain applications.

- We can also augment data by manipulating the feature space and generate new data samples with similar features. Representative method include SMOTE [3] and Autoencoder [15].

- One recently popular method is based on adversarial models. Goodfellow et al. proposed adversarial training first [10]. Following adversarial training, Goodfellow et al. further proposed generative adversarial networks (GAN) [9]. GAN has been successfully used on image [9], natural language processing [47], and reinforcement learning [13]. Recently, GANs has been used in few-shot learning [8].

- Unlabelled data can also be used to help data augmentation.

- Semi-supervised learning uses unlabeled data in learning process.

* Co-training [2] creates two models by using different features and the testing samples are iteratively added into the training data of one model if they are assigned with a class with high confidence by the other model.

* Graph-based methods [51] defines the objective function by the prediction and also by the difference of predicted values of "similar" data samples, where similar is defined by other similarity metrics.

* Self-training methods uses kNN propagation and pseudolabeling [17].

* A recently proposed effective method MixMatch [1] combines self-training, entropy minimization, consistency regularization $[16,33]$.

- Active learning [29] enables human in the loop and selects the data samples for human to label, where those data samples can help the most in defining the decision boundaries.

\section{RELEVANCE TO THE COMMUNITY}

The research community of web search and data mining cares about how to use machine learning techniques in the real-world problems. In the real world, however, we often face small-data challenges. The community seeks how to innovate the techniques so that the methods are more applicable in the real world. Many related approaches have been approached while there is still a great room for improvement. Giving this tutorial helps our community understand the state of the art and can better innovate the techniques in this topic as well.

\section{REFERENCES TO RELATED RESOURCES}

- CVPR 2019 Tutorial: Meta-Learning for Computer Vision (https://metalearning-cvpr2019.github.io/)
- ICML 2019 Tutorial: Meta-Learning: from Few-Shot Learning to Rapid Reinforcement Learning (https://sites.google.com/ view/icml19metalearning)

- CVPR 2018 Tutorial: GANs (https://sites.google.com/view/ cvpr2018tutorialongans/)

As we can see that, most related tutorials have been offered at computer vision and machine learning conferences, where they mostly focus on the application in vision or on the pure learning techniques. Our tutorial is different where we focus on a more comprehensive techniques in learning with small data and their applications in real-world problems other than computer vision.

\section{FORMAT AND DETAILED SCHEDULE}

This will be a half-day 3-hour tutorial. Before the break, we will talk about Part I on knowledge transfer from learned models in the source domain. After the break, we will talk about transfer knowledge from external domain knowledge and how to augment data.

- Introduction (20 minutes)

- Real-world applications with small data.

- Unique challenges of learning with small data.

- Part I: Transfer knowledge from models (70 minutes)

- Transfer learning

- Multi-task Learning

- Meta-learning

- Coffee break

- Part II: Transfer knowledge from domain expert (45 minutes)

- Enriching representations using knowledge graph

- Regularizing the loss function by incorporating domain knowledge

- Part III: Data augmentation (35 minutes)

- Augmentation using labeled data

* Hand-crafted rule based augmentation (application specific techniques)

* Feature space augmentation

* Adversarial models

- Augmentation using unlabeled data

* Semi-supervised learning

* Active learning

- Conclusion and Future Outlook (10 minutes)

\section{TYPE OF SUPPORT MATERIALS TO BE SUPPLIED TO ATTENDEES}

Each presenter will bring their own laptop. The equipment to be needed are projector, power socket, and laptop adapters. The attendees are not required to bring any equipment.

\section{ACKNOWLEDGMENTS}

The work was supported in part by NSF awards \#1652525 and \#1618448. The views and conclusions contained in this paper are those of the authors and should not be interpreted as representing any funding agencies. 


\section{REFERENCES}

[1] David Berthelot, Nicholas Carlini, Ian Goodfellow, Nicolas Papernot, Avital Oliver, and Colin Raffel. 2019. Mixmatch: A holistic approach to semi-supervised learning. arXiv preprint arXiv:1905.02249 (2019).

[2] Avrim Blum and Tom Mitchell. 1998. Combining labeled and unlabeled data with co-training. In Proceedings of the eleventh annual conference on Computational learning theory. Citeseer, 92-100.

[3] Nitesh V Chawla, Kevin W Bowyer, Lawrence O Hall, and W Philip Kegelmeyer 2002. SMOTE: synthetic minority over-sampling technique. Fournal of artificial intelligence research 16 (2002), 321-357.

[4] Edward Choi, Mohammad Taha Bahadori, Le Song, Walter F Stewart, and Jimeng Sun. 2017. GRAM: graph-based attention model for healthcare representation learning. In Proceedings of the 23rd ACM SIGKDD International Conference on Knowledge Discovery and Data Mining. ACM, 787-795.

[5] Chelsea Finn, Pieter Abbeel, and Sergey Levine. 2017. Model-Agnostic MetaLearning for Fast Adaptation of Deep Networks. In ICML. 1126-1135.

[6] Chelsea Finn and Sergey Levine. 2018. Meta-learning and universality: Deep representations and gradient descent can approximate any learning algorithm. In ICLR.

[7] Chelsea Finn, Kelvin Xu, and Sergey Levine. 2018. Probabilistic Model-Agnostic Meta-Learning. In NeurIPS.

[8] Hang Gao, Zheng Shou, Alireza Zareian, Hanwang Zhang, and Shih-Fu Chang. 2018. Low-shot learning via covariance-preserving adversarial augmentation networks. In Advances in Neural Information Processing Systems. 975-985.

[9] Ian Goodfellow, Jean Pouget-Abadie, Mehdi Mirza, Bing Xu, David Warde-Farley, Sherjil Ozair, Aaron Courville, and Yoshua Bengio. 2014. Generative adversarial nets. In Advances in neural information processing systems. 2672-2680.

[10] Ian J Goodfellow, Jonathon Shlens, and Christian Szegedy. 2014. Explaining and harnessing adversarial examples. arXiv preprint arXiv:1412.6572 (2014).

[11] Jiatao Gu, Yong Wang, Yun Chen, Kyunghyun Cho, and Victor OK Li. 2018 Meta-learning for low-resource neural machine translation. In EMNLP.

[12] Xu Han, Zhiyuan Liu, and Maosong Sun. 2018. Neural knowledge acquisition via mutual attention between knowledge graph and text. In Thirty-Second AAAI Conference on Artificial Intelligence.

[13] Jonathan Ho and Stefano Ermon. 2016. Generative adversarial imitation learning. In Advances in neural information processing systems. 4565-4573.

[14] Zhiting Hu, Xuezhe Ma, Zhengzhong Liu, Eduard Hovy, and Eric Xing. 2016. Harnessing Deep Neural Networks with Logic Rules. In Proceedings of the 54th Annual Meeting of the Association for Computational Linguistics (Volume 1: Long Papers). 2410-2420.

[15] Diederik P Kingma and Max Welling. 2013. Auto-encoding variational bayes. arXiv preprint arXiv:1312.6114 (2013).

[16] Samuli Laine and Timo Aila. 2016. Temporal ensembling for semi-supervised learning. arXiv preprint arXiv:1610.02242 (2016).

[17] Dong-Hyun Lee. 2013. Pseudo-label: The simple and efficient semi-supervised learning method for deep neural networks. In Workshop on Challenges in Representation Learning, ICML, Vol. 3. 2.

[18] Yoonho Lee and Seungjin Choi. 2018. Gradient-based meta-learning with learned layerwise metric and subspace. In ICML. 2933-2942.

[19] Christy Y Li, Xiaodan Liang, Zhiting Hu, and Eric P Xing. 2019. Knowledge-driven encode, retrieve, paraphrase for medical image report generation. In Thirty-Third AAAI Conference on Artificial Intelligence.

[20] Zheng Li, Xin Li, Ying Wei, Lidong Bing, Yu Zhang, and Qiang Yang. 2019. Transferable End-to-End Aspect-based Sentiment Analysis with Selective Adversarial Learning. (2019).

[21] Mingsheng Long, Yue Cao, Jianmin Wang, and Michael I Jordan. 2015. Learning transferable features with deep adaptation networks. In ICML.

[22] Mingsheng Long, Zhangjie Cao, Jianmin Wang, and S Yu Philip. 2017. Learning multiple tasks with multilinear relationship networks. In Advances in neural information processing systems. 1594-1603.

[23] Mingsheng Long, Han Zhu, Jianmin Wang, and Michael I Jordan. 2017. Deep transfer learning with joint adaptation networks. In Proceedings of the 34th International Conference on Machine Learning-Volume 70. JMLR. org, 2208-2217.

[24] Fenglong Ma, Jing Gao, Qiuling Suo, Quanzeng You, Jing Zhou, and Aidong Zhang. 2018. Risk prediction on electronic health records with prior medical knowledge. In Proceedings of the 24th ACM SIGKDD International Conference on Knowledge Discovery \& Data Mining. ACM, 1910-1919.

[25] Fenglong Ma, Yaqing Wang, Houping Xiao, Ye Yuan, Radha Chitta, Jing Zhou, and Jing Gao. 2018. A General Framework for Diagnosis Prediction via Incorporating Medical Code Descriptions. In 2018 IEEE International Conference on
Bioinformatics and Biomedicine (BIBM). IEEE, 1070-1075.

[26] Fenglong Ma, Quanzeng You, Houping Xiao, Radha Chitta, Jing Zhou, and Jing Gao. 2018. Kame: Knowledge-based attention model for diagnosis prediction in healthcare. In Proceedings of the 27th ACM International Conference on Information and Knowledge Management. ACM, 743-752.

[27] Boris Oreshkin, Pau Rodríguez López, and Alexandre Lacoste. 2018. Tadam: Task dependent adaptive metric for improved few-shot learning. In NeurIPS. 721-731.

[28] Sebastian Ruder. 2017. An overview of multi-task learning in deep neural networks. arXiv preprint arXiv:1706.05098 (2017).

[29] Burr Settles. 2009. Active learning literature survey. Technical Report. University of Wisconsin-Madison Department of Computer Sciences.

[30] Jake Snell, Kevin Swersky, and Richard Zemel. 2017. Prototypical networks for few-shot learning. In NeurIPS. 4077-4087.

[31] Robert Speer, Joshua Chin, and Catherine Havasi. 2017. Conceptnet 5.5: An open multilingual graph of general knowledge. In Thirty-First AAAI Conference on Artificial Intelligence.

[32] Xianfeng Tang, Yandong Li, Yiwei Sun, Huaxiu Yao, Prasenjit Mitra, and Suhang Wang. 2019. Robust graph neural network against poisoning attacks via transfer learning. arXiv preprint arXiv:1908.07558 (2019).

[33] Antti Tarvainen and Harri Valpola. 2017. Mean teachers are better role models: Weight-averaged consistency targets improve semi-supervised deep learning results. In Advances in neural information processing systems. 1195-1204.

[34] Kristina Toutanova, Danqi Chen, Patrick Pantel, Hoifung Poon, Pallavi Choudhury, and Michael Gamon. 2015. Representing text for joint embedding of text and knowledge bases. In Proceedings of the 2015 Conference on Empirical Methods in Natural Language Processing. 1499-1509.

[35] Eric Tzeng, Judy Hoffman, Kate Saenko, and Trevor Darrell. 2017. Adversarial discriminative domain adaptation. In CVPR.

[36] Eric Tzeng, Judy Hoffman, Ning Zhang, Kate Saenko, and Trevor Darrell. 2014. Deep domain confusion: Maximizing for domain invariance. arXiv preprint arXiv:1412.3474 (2014).

[37] Oriol Vinyals, Charles Blundell, Timothy Lillicrap, Daan Wierstra, et al. 2016. Matching networks for one shot learning. In NeurIPS. 3630-3638.

[38] Risto Vuorio, Shao-Hua Sun, Hexiang Hu, and Joseph J Lim. 2018. Toward multimodal model-agnostic meta-learning. arXiv preprint arXiv:1812.07172 (2018).

[39] Yaqing Wang and Quanming Yao. 2019. Few-shot learning: A survey. arXiv preprint arXiv:1904.05046 (2019)

[40] Ying Wei, Peilin Zhao, Huaxiu Yao, and Junzhou Huang. 2019. Transferable Neural Processes for Hyperparameter Optimization. arXiv preprint arXiv:1909.03209 (2019).

[41] Huaxiu Yao, Yiding Liu, Ying Wei, Xianfeng Tang, and Zhenhui Li. 2019. Learning from Multiple Cities: A Meta-Learning Approach for Spatial-Temporal Prediction. In The World Wide Web Conference. ACM, 2181-2191.

[42] Huaxiu Yao, Ying Wei, Junzhou Huang, and Zhenhui Li. 2019. Hierarchically Structured Meta-learning. In ICML. 7045-7054.

[43] Huaxiu Yao, Chuxu Zhang, Ying Wei, Meng Jiang, Suhang Wang, Junzhou Huang, Nitesh V Chawla, and Zhenhui Li. 2020. Graph Few-shot Learning via Knowledge Transfer. AAAI Conference on Artificial Intelligence (2020).

[44] Changchang Yin, Rongjian Zhao, Buyue Qian, Xin Lv, and Ping Zhang. [n. d.]. Domain Knowledge Guided Deep Learning with Electronic Health Records.

[45] Jaesik Yoon, Taesup Kim, Ousmane Dia, Sungwoong Kim, Yoshua Bengio, and Sungjin Ahn. 2018. Bayesian Model-Agnostic Meta-Learning. In NIPS. 7343-7353.

[46] Sung Whan Yoon, Jun Seo, and Jaekyun Moon. 2019. TapNet: Neural Network Augmented with Task-Adaptive Projection for Few-Shot Learning. In ICML.

[47] Lantao Yu, Weinan Zhang, Jun Wang, and Yong Yu. 2017. Seqgan: Sequence generative adversarial nets with policy gradient. In Thirty-First AAAI Conference on Artificial Intelligence.

[48] Xinshi Zang, Huaxiu Yao, Guanjie Zheng, Nan Xu, Kai Xu, and Zhenhui Li. 2020. MetaLight: Value-based Meta-reinforcement Learning for Online Universal Traffic Signal Control. AAAI Conference on Artificial Intelligence (2020).

[49] Chuxu Zhang, Huaxiu Yao, Chao Huang, Meng Jiang, Zhenhui Li, and Nitesh V Chawla. 2020. Few-shot Knowledge Graph Completion. AAAI Conference on Artificial Intelligence (2020).

[50] Jiacheng Zhang, Yang Liu, Huanbo Luan, Jingfang Xu, and Maosong Sun. 2017. Prior Knowledge Integration for Neural Machine Translation using Posterior Regularization. In Proceedings of the 55th Annual Meeting of the Association for Computational Linguistics (Volume 1: Long Papers). 1514-1523.

[51] Xiaojin Zhu and Zoubin Ghahramani. 2002. Learning from labeled and unlabeled data with label propagation. Technical Report. Citeseer. 